\title{
A research on the preparation of oil-adsorbing hydrophobic porous resins by high internal phase emulsions (HIPEs) template
}

https://doi.org/10.1515/secm-2019-0015

Received August 9, 2017; accepted January 25, 2019

\begin{abstract}
In this work, hierarchical porous resins (PRs) are fabricated and applied as novel adsorbent for removal of oily substance. The oil/water (o/w) high internal phase emulsions (HIPEs), stabilized by phenolic resin precursor and tween 80, were used as the template for simultaneous polymerization of aqueous phase, methenamine, sodium persulfate and liquid paraffin. Subsequently, dopamine hydrochloride, 1-dodecanethiol and $\mathrm{Fe}_{3} \mathrm{O}_{4}$ particles are grafted onto the interface of PRs via adhesion of dopamine and Markel addition reaction between quinone groups on dopamine and sulfhydryl groups on 1-dodecanethiol. The as-prepared composites possessed hydrophobicity and magnetic property (HPRs). The physical and chemical properties, morphology and adsorption properties of HPRs are characterized by XRD, FT-IR, TGA, SEM, $\mathrm{N}_{2}$ adsorptiondesorption isotherms. The as-synthesized HPRs composites had a typical hierarchical porous structure, and the resulted macropores with a well-defined open-cell and interconnecting pore throat structure could be controlled via the composition of the aqueous phase of emulsion. The hierarchical porous structure of HPRs contained macropores ranging from $0.3 \mu \mathrm{m}$ to $4 \mu \mathrm{m}$ and mesopores of $11.26 \mathrm{~nm}$. HPRs have rough and hydrophobic surface (water contact angle of $130^{\circ}$ ) with specific surface area of $15.06 \mathrm{~m}^{2} / \mathrm{g}$ and pore volume of $0.0349 \mathrm{~cm}^{3} / \mathrm{g}$. The oil adsorption rate and the oil retention rate for toluene are $11.765 \mathrm{~g} / \mathrm{g}$ and $86.43 \%$, respectively. The oil adsorption rate in the first minute can reach more than $80 \%$ of the highest oil adsorption rate. The oil adsorption rate of HPRs after three recycling experiments can still reach $87.32 \%$ of the first-time oil adsorption rate.
\end{abstract}

Keywords: porous adsorption material; high internal phase emulsion; oil-adsorbing; polydopamine; ferriferrous oxide

Xiazhi Zhou: School of metallurgy and materials engineering, Jiangsu University of Science and Technology, Zhangjiagang 215600, China

These authors equally contributed to the work.

\section{Introduction}

Nowadays, with the rapid development of petrochemical and transportation industries, environmental pollution problems caused by oil spilling accidents and oily sewage disposal make a serious threat to ecosystem and human life. Thus, there is an urgent requirement to find fast and effective methods to control oily substances pollution. Conventional oil adsorbing materials adsorb oily substances on the surface or within the capillaries, which have disadvantages such as small oil adsorption capacity, poor oil retention performance, and slow adsorption rate. The development of new efficient oil adsorbing materials has gained more attention [1].

In recent years, a large number of oil adsorbing materials have been developed and studied, including surface hydrophobic modification of hydrophobic lipophilic sponge [2], nanofiber [3], porous nitrocellulose [4], modified metal net [5] and graphene bubble [6]. Oil adsorbing

Wei Zhou: School of Environmental and Chemical Engineering, Shanghai University, 99 Shangda Road, Shanghai 200444, China Dianchun Ju: School of metallurgy and materials engineering, Jiangsu University of Science and Technology, Zhangjiagang 215600, China

Suzhou Institute of science and technology, Jiangsu University of Science and Technology, Zhangjiagang 215600, China

*Corresponding Author: Yinxian Peng: School of metallurgy and materials engineering, Jiangsu University of Science and Technology, Zhangjiagang 215600, China

Suzhou Institute of science and technology, Jiangsu University of Science and Technology, Zhangjiagang 215600, China

School of Environmental and Chemical Engineering, Jiangsu University of Science and Technology, Zhenjiang 212003, China, E-mail: pyxhx@just.edu.cn

Lei Zhou: School of metallurgy and materials engineering, Jiangsu University of Science and Technology, Zhangjiagang 215600, China Sheng Tang: School of Environmental and Chemical Engineering, Jiangsu University of Science and Technology, Zhenjiang 212003, China

*Corresponding Author: Jing Wang: School of Environmental and Chemical Engineering, Jiangsu University of Science and Technology, Zhenjiang 212003, China, E-mail: wangjingalice1@yeah.net 
porous polymers have attracted more attention because of their light quality, high strength, large specific surface area and huge adsorption capacity. Atta fabricated porous polymer oil absorbents from non-woven polyethylene terephthalate fibers modified by the adherence of crosslinked polymer coatings to the fiber surface, which showed higher porosity and a high sorption capacity [7]. The porous polymers can be synthesized by direct synthesis method [8], interfacial polymerization, breathing figure method [10], emulsion polymerization [11] and high internal phase emulsions (HIPEs) template method [12-14].

The HIPEs polymerization is mostly applied for the synthesis of zwitterionic hydrogels $[15,16]$ and so on, but rarely reported in the oil and water separation, which is an area that can be explored. Eissa and Cameron described the preparation of highly porous monolithic polymers (poly-HIPEs) by emulsion templating and thiol-ene/yne photopolymerisation. Issues associated with monomer solubility and/or stability of the produced high internal phase emulsion are solved [17]. In the poly-HIPEs, porous poly samples with total porosity up to $91 \%$, porous polyHIPEs monoliths have open cellular morphology with cavity diameters between sub- $\mu \mathrm{m}$ and 30- $\mu \mathrm{m}$ [18].

In a general way, the hydrophilic property of the polymers synthesized by HIPEs depends on the monomers of materials, and an increase in hydrophobicity of adsorbent will also enhance the adsorption capability of materials for oil contaiminations [19]. Materials polymerized from the w/o emulsion where hydrophobic monomer dissolved in the oil acts as continuous phase and the water disperses in it are commonly hydrophobic. Hydrophilic polyHIPEs were produced by adding hydrophilic monomers to the HIPE's internal phase in w/o HIPEs [20, 21].

In this paper, porous resins (PRs) were synthesized by HIPEs with phenolic resin precursor, methenamine, sodium persulfate, tween 80 and liquid paraffin. Then hydrophobic porous phenolic resins (HPRs) were prepared by modifying PRs with dopamine hydrochloride, 1dodecanethiol and $\mathrm{Fe}_{3} \mathrm{O}_{4}$ particles to achieve hydrophobicity and magnetic property. $\mathrm{Fe}_{3} \mathrm{O}_{4}$ particles were adhered on the surface of the materials by the strong adhesion effect of polydopamine, which makes the materials easy to recycle. The hydrophobicity of PRs was improved by 1-dodecanethiol which reacts with the dopamine to render hydrophobicity to the surface of the PRs. The XRD, FTIR, TGA, SEM, $\mathrm{N}_{2}$ adsorption-desorption isotherms were used to characterize the morphology, physical and chemical properties and adsorption properties of the products.

\section{Materials and methods}

\subsection{Materials}

Phenol, formaldehyde, liquid paraffin, methenamine, sodium persulfate, tween 80 , dopamine hydrochloride, 1-dodecanethiol and $\mathrm{Fe}_{3} \mathrm{O}_{4}$ nanoparticles, ferric chloride, petroleum ether $30-60^{\circ} \mathrm{C}$, toluene, cyclohexane and acetone were supplied from Aladdin reagent Co., Ltd (Shanghai, China). Bean oil was purchased from a local supermarket. Engine oil and gasoline were bought from a local gas station. Deionized water was used for all experiments.

\subsection{Instruments}

HJ-6A magnetic stirrer, H-1650 high-speed centrifuge, KQ5200DE ultrasonic machine, Nicolet NEXUS-870 FTIR apparatus (U.S.A.), XRD-6000 (Shimadzu), ASAP2020 physical adsorption instrument (U.S.A.), JSM-6480 scanning electron microscope (SEM, Japan), Pyris Diamond thermo-gravimetric analyzer (U.S.A.), optical contact angle measurement instrument (KSV CM200), NORAN System SIX spectrometer (EDS, U.S.A.).

\subsection{Preparation of hydrophobic porous phenolic resins (HPRs)}

\subsubsection{Preparation of $\mathrm{Fe}_{3} \mathrm{O}_{4}$ particles}

$\mathrm{FeCl}_{3} \cdot 6 \mathrm{H}_{2} \mathrm{O}(0.675 \mathrm{~g})$ was added into ethylene glycol $(20 \mathrm{~mL})$, which was stirred at the speed of $800 \mathrm{rpm}$ until a transparent state was obtained. Subsequently, sodium acetate $(1.8 \mathrm{~g})$ and polyethylene glycol $(0.5 \mathrm{~g})$ were added into the above solution. The mixture was stirred with a magnetic stirring at the speed of $800 \mathrm{rpm}$ for $30 \mathrm{~min}$. Then the solution was transferred

into a reaction kettle $(25 \mathrm{~mL})$, and reacted at $180^{\circ} \mathrm{C}$ for $16 \mathrm{~h}$. After the reaction kettle was

naturally cooled to room temperature, black precipitate was obtained by magnetic separation. Then the precipitate was purified with anhydrous ethanol for 3-5 times. Finally, $\mathrm{Fe}_{3} \mathrm{O}_{4}$ particles were obtained by drying the precipitate at $60^{\circ} \mathrm{C}[22]$.

\subsubsection{Preparation of phenolic resin precursor}

Phenol (7.2 g) was added into sodium hydroxide solution $(12 \mathrm{~mL}, 0.02 \mathrm{~g} / \mathrm{mL})$. Then the above solution was trans- 
ferred in a round bottom flask stirred with a magnetic stirrer at a speed of $400 \mathrm{rpm}$ at $43^{\circ} \mathrm{C}$ for $10 \mathrm{~min}$. Finally, formaldehyde solution ( $15 \mathrm{~mL}, 37 \%$ ) was added, and the mixture was reacted at $73^{\circ} \mathrm{C}$ for one hour. Phenolic resin precursor was achieved after cooling to room temperature.

\subsubsection{Preparation of porous resins (PRs)}

Methenamine $(0.15 \mathrm{~g})$ and sodium persulfate $(0.01 \mathrm{~g})$ were added to the solution of phenolic resin precursor $(5 \mathrm{~mL}$, $\mathrm{pH}=7.0$ ) under stirring. After stirring at the speed of 600 rpm for $5 \mathrm{~min}$, the solution was transferred to flask with three necks $(250 \mathrm{~mL})$. Then tween $80(0.2 \mathrm{~g})$ was added under stirring at the speed of $800 \mathrm{rpm}$ to the solution and the stirring was continued for $5 \mathrm{~min}$. After decreasing the stirring speed to $400 \mathrm{rpm}$, a certain amount of liquid paraffin (according to the proportion of continuous phase to dispersed phase) was added into the above solution at the rate of two drops per second. Then the obtained emulsion was transferred to the mould, sealed and heated in the water bath at $80^{\circ} \mathrm{C}$ for $24 \mathrm{~h}$. The polymerization product was purified by Soxhlet extraction with petroleum ether 30$60^{\circ}$ to remove liquid paraffin, surface active agent, non reactive monomer and other impurities. The porous resins (PRs) were obtained after drying for $24 \mathrm{~h}$ at $60^{\circ}$.

\subsubsection{The preparation of hydrophobic porous resins (HPRs)}

Because of the strong adhesion of polydopamine, the $\mathrm{Fe}_{3} \mathrm{O} 4$ particles were adhered on the surface of PRs by polydopamine. The obtained product became magnetic, which makes it convenient to recycle under magnetic field. In alkaline condition, the catechol groups were rich in polydopamine and were easily oxidized to quinoid structure. Then, the hydrophobic material was obtained by michael addition reaction [23].

Orthogonal test $\mathrm{L}_{9}\left(3_{4}\right)$ was used in order to optimize the technological parameters of the high performance oil adsorbing material. Independent variable factors included water-oil ratio (the volume ratio of continuous phase to dispersed phase), the dosages of 1-dodecanethiol, dopamine hydrochloride and $\mathrm{Fe}_{3} \mathrm{O}_{4}$ particles. Factors and levels of orthogonal experiment are shown in Table 1.

A certain amount of dopamine hydrochloride was dissolved in ethanol aqueous solution $(50 \mathrm{~mL}, 1: 1(\mathrm{~V} / \mathrm{V}))$, and then the 1-dodecanethiol was added. After the solution was adjusted to $\mathrm{pH} 8.0$ with sodium hydroxide solution or hydrochloric acid solution, $\mathrm{Fe}_{3} \mathrm{O}_{4}$ particles were added. After 10 min's ultrasonic dispersion, PRs (1.00 g) was added. The solution was stirred at the speed of 600 rpm for $12 \mathrm{~h}$ (room temperature). The product was washed by deionized water and ethanol for 3 times respectively, and finally was dried at $60^{\circ} \mathrm{C}$ for $24 \mathrm{~h}$ to obtain hydrophobic porous oil adsorption resin (HPRs).

\subsection{Characterization of HPRs}

The morphology of the PRs and HPRs was examined with a scanning electron microscope (SEM, JSM-6480, JEOL, Japan) and element analysis was carried out by elemental analyzer. The crystal structures of the products were characterized by X-ray diffraction (XRD) analysis (Ulitima IV, Japan). Radial scans were recorded in the reflection scanning mode from $2 \theta=10-90^{\circ}$ with a scanning rate of $6^{\circ} / \mathrm{min}$. The Brunauer-Emmett-Teller (BET) surface area and pore structure of HPRs were determined by a Micromeritics ASAP 2020 volumetric adsorption analyzer. FTIR analysis was carried out in the range of $400-4000 \mathrm{~cm}^{-1}$ using a FT-IR spectrophotometer (Thermo Nicolet, NEXUS, TM)

in $\mathrm{KBr}$ pellets. The thermal stability of HPRs was tested by the thermal gravimetric analysis (TGA), and the powder sample of HPRs (about $5 \mathrm{mg}$ ) was heated with a scanning rate of $15^{\circ} \mathrm{C} / \mathrm{min}$ from room temperature to $800^{\circ}$ under $\mathrm{N}_{2}$ atmosphere. The contact angles of PRs and HPRs were measured through the Optical Contact Angle Measuring Device (KSV CM200), characterizing the changes of the water angle of PRs, HPRs and recycled HPRs.

\subsection{The performance test}

\subsubsection{Oil adsorption rate}

A certain amount of samples of HPRs $\left(m_{0}\right)$ was added to oil or organic solvents for adsorption at a certain temperature. Then the samples were taken out after a certain period of time. After dripping for $5 \mathrm{~min}$ the weight of the samples $\left(\mathrm{m}_{1}\right)$ was measured. According to the formula (1) the oil adsorption rate $\mathrm{Q}$ of the material is calculated [24].

$$
Q=\frac{m_{1}-m_{0}}{m_{0}}
$$

\subsubsection{Oil retention rate}

A certain amount of samples of HPRs $\left(m_{0}\right)$ was added to the oil for adsorption, and after adsorption saturation, the 
Table 1: Factors and levels of orthogonal experiment

\begin{tabular}{clrcc}
\hline Factors & $A$ & $B(\mu \mathrm{L} / \mathrm{g})$ & $\mathrm{C}(\mathrm{mg} / \mathrm{g})$ & $\mathrm{D}(\mathrm{mg} / \mathrm{g})$ \\
\hline 1 & $1: 3$ & 20 & 50 & 100 \\
2 & $1: 4$ & 30 & 75 & 200 \\
3 & $1: 5$ & 40 & 100 & 300
\end{tabular}

Note: The dosage of 1-dodecanethiol, dopamine hydrochloride and $\mathrm{Fe}_{3} \mathrm{O}_{4}$ particles were added compared with the mass of PRs. A, B, C and D stand for water-oil ratio, the dosage of 1-dodecanethiol, the dosage of dopamine hydrochloride and the dosage of $\mathrm{Fe}_{3} \mathrm{O}_{4}$ particles respectively.

weight of samples $\left(m_{1}\right)$ was measured. Next, the samples were transferred to centrifuge tube and centrifuged at a rate of $1000 \mathrm{r} / \mathrm{min}$ for $5 \mathrm{~min}$ and the weight of the samples $\left(\mathrm{m}_{2}\right)$ was measured. According to the formula (2) the oil retention rate $\mathrm{K}$ of the material is calculated [25].

$$
K=\frac{m_{2}-m_{0}}{m_{1}-m_{0}}
$$

\subsubsection{Recycling efficiency}

Hydrophobic oil adsorbing materials were purified by Soxhlet extraction with petroleum ether for $12 \mathrm{~h}$ to remove the adsorbed oily substances. Recycled HPRs was obtained after drying the materials for $24 \mathrm{~h}$ and the oil adsorption rate was measured again to evaluate the adsorption capability of the used HPRs.

\section{Results and discussion}

\subsection{Orthogonal test analysis}

Since various factors potentially influenced the adsorption properties of the synthesized HPRs, the optimization of the synthesis condition was critical for enhancing the efficiency of adsorption. In this study, orthogonal test L9 (34) was used to optimize the reaction condition. The results of orthogonal test are shown in Table 2. It can be known from the Table 2 that the influencing factors of the adsorption properties of HPRs were water-oil ratio, the dosage of 1dodecanethiol, the dosage of dopamine hydrochloride and the dosage of $\mathrm{Fe}_{3} \mathrm{O} 4$ particles in descending order. The optimum condition is $\mathrm{A}_{3} \mathrm{~B} 3 \mathrm{C} 3 \mathrm{D} 2$ 2: water-oil ratio was 1:5, the dosages of 1-dodecanethiol $40 \mu \mathrm{L} / \mathrm{g}$, dopamine hydrochloride $10 \%$, Fe304 particles $20 \%$.

\subsection{X-ray diffraction analysis (XRD)}

The $\mathrm{Fe}_{3} \mathrm{O}_{4}$ particles prepared by the solvothermal method are black powders, and the XRD pattern of $\mathrm{Fe}_{3} \mathrm{O}_{4}$ particles is presented in Fig. 1. As it can be seen from Fig. 1, the prepared $\mathrm{Fe}_{3} \mathrm{O}_{4}$ particles have sharp diffraction peaks and the angle $2 \theta$ of the diffraction peak of samples were at $30.06^{\circ}$, $35.40^{\circ}, 42.96^{\circ}, 53.42^{\circ}, 56.86^{\circ}, 62.46^{\circ}$ and $73.92^{\circ}$, which are corresponding to the crystal face (220), (311), (400), (422), (511), (440), (533) of $\mathrm{Fe}_{3} \mathrm{O}_{4}$ crystal respectively. The position and relative intensity of all the diffraction peaks match well with the standard PDF card (JCPDS 19-0629) of magnetite $\mathrm{Fe}_{3} \mathrm{O}_{4}$ with inverse spinel structure. The products are monophasic and there is no impurity phase.

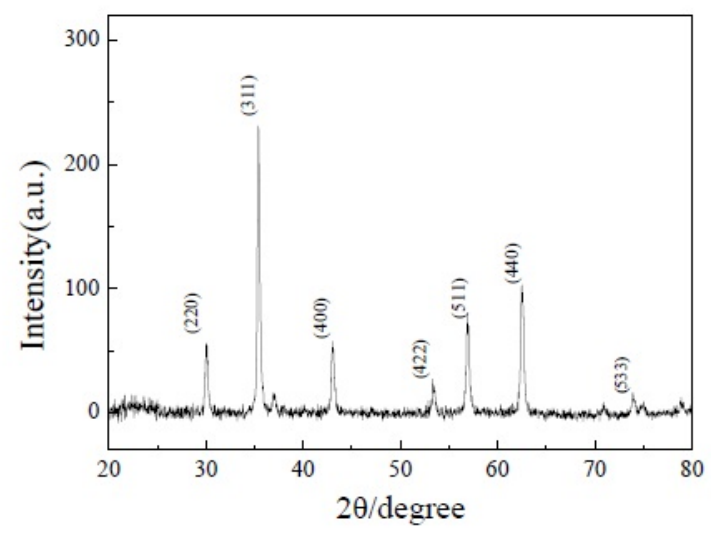

Figure 1: The XRD spectrum of $\mathrm{Fe}_{3} \mathrm{O}_{4}$ particles

\subsection{Infrared spectrum analysis (FT-IR)}

FT-IR was used to study the functional groups of PRs as well as HPRs. The FT-IR spectra of PRs (a) and HPRs (b) are shown in Fig. 2. The broad adsorption peak at $3420 \mathrm{~cm}^{-1}$ corresponds to the stretching vibration peak of phenolic hydroxyl and the stretching vibration peak of amino N$\mathrm{H}$ bond. The adsorption peak at $3010 \mathrm{~cm}^{-1}, 2920 \mathrm{~cm}^{-1}$ and $2870 \mathrm{~cm}^{-1}$ is attributed to the $\mathrm{C}-\mathrm{H}$ stretching vibration peak. The adsorption peaks around $1600 \mathrm{~cm}^{-1}$ and $1480 \mathrm{~cm}^{-1}$ are the mixed peaks of the skeletal vibration of benzene ring and vibration adsorption in-plane of amino. The twisted vibration peak of methylene is at $1360 \mathrm{~cm}^{-1}$ 
Table 2: Results of orthogonal experiment

\begin{tabular}{rrrrlc}
\hline No. & $\mathrm{A}$ & $\mathrm{B}(\mu \mathrm{L} / \mathrm{g})$ & $\mathrm{C}(\mathrm{mg} / \mathrm{g})$ & $\mathrm{D}(\mathrm{mg} / \mathrm{g})$ & oil(toluene) adsorption rate/g/g \\
\hline 1 & $1: 3$ & 20 & 50 & 100 & 10.153 \\
2 & $1: 3$ & 30 & 75 & 200 & 10.694 \\
3 & $1: 3$ & 40 & 100 & 300 & 10.973 \\
4 & $1: 4$ & 20 & 75 & 300 & 10.987 \\
5 & $1: 4$ & 30 & 100 & 100 & 11.268 \\
6 & $1: 4$ & 40 & 50 & 200 & 11.464 \\
7 & $1: 5$ & 20 & 100 & 200 & 11.394 \\
8 & $1: 5$ & 30 & 50 & 300 & 11.452 \\
9 & $1: 5$ & 40 & 75 & 100 & 11.765 \\
$\mathrm{k} 1$ & 10.607 & 10.845 & 11.023 & 11.062 & \\
$\mathrm{k} 2$ & 11.240 & 11.138 & 11.149 & 11.184 & \\
$\mathrm{k} 3$ & 11.537 & 11.401 & 11.212 & 11.137 & \\
$\mathrm{R}$ & 0.930 & 0.556 & 0.189 & 0.122 & \\
\hline
\end{tabular}

Note: $\mathrm{K}$ is average, and $\mathrm{R}$ is extreme difference. The dosage of 1-dodecanethiol, dopamine hydrochloride and $\mathrm{Fe}_{3} \mathrm{O}_{4}$ particles were added compared with the mass of PRs. A, B, C and D stand for water-oil ratio, the dosage of 1-dodecanethiol, the dosage of dopamine hydrochloride and the dosage of $\mathrm{Fe}_{3} \mathrm{O}_{4}$ particles respectively.

and the stretching vibration peak of $\mathrm{C}-\mathrm{O}$ bond in phenol is at $1230 \mathrm{~cm}^{-1}$. Several absorption peaks near $1060 \mathrm{~cm}^{-1}$ are peaks of $\mathrm{C}-\mathrm{N}$. The $\mathrm{N}-\mathrm{H}$ surface bending vibration peak is at $670 \mathrm{~cm}^{-1}$. PRs have a weak vibration peak of N-H due to the presence of $\mathrm{N}-\mathrm{H}$ groups in the crosslinking agent methenamine. However, in comparison with PRs, the N$\mathrm{H}$ vibration peak of HPRs is stronger than that of PRs. The reason is that during the modification reaction, dopamine hydrochloride molecules take place self-polymerization to produce polydopamine and the obtained polydopamine molecules, which cover on the surfaces of the materials to strengthen the out-of-plane bending vibration peak of $\mathrm{N}$ $\mathrm{H}$.

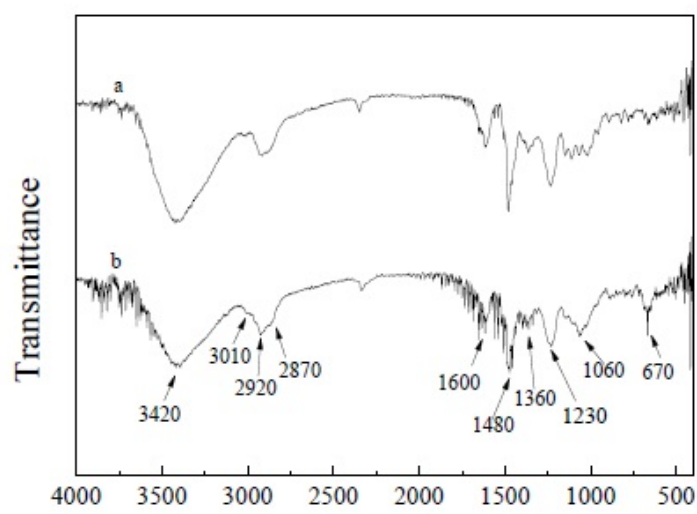

Wavenumbers $\left(\mathrm{cm}^{-1}\right)$

\subsection{Thermal gravimetric analysis (TGA)}

The TGA curves of PRs and HPRs are shown in Fig. 3. It can be seen from the figure that the thermal weight loss process of PRs and HPRs can be divided into four stages. The loss from room temperature to $160^{\circ} \mathrm{C}$ is due to the loss of water or small molecules, and the loss of PRs and HPRs are both $c a$. $2.5 \%$. From $160^{\circ} \mathrm{C}$ to $330^{\circ} \mathrm{C}$, the weight loss is due to the further crosslinking of the resin, the condensation reaction of hydroxymethyl derivatives or the removal of hydroxymethyl groups, which release water, formaldehyde, phenol and so on. At this stage, the crosslinking density of the resin is the biggest and the structure of the resin is stable, and the loss of PRs and HPRs are both $c a .15 \%$. From $330^{\circ} \mathrm{C}$ to $600^{\circ} \mathrm{C}$, the weight loss ratios of PRs and HPRs are about $45 \%$ and $37 \%$, respectively. The decomposition, cyclization, aromatization, condensation reaction and other chemical reactions lead to the significant weight loss and release a large number of $\mathrm{H}_{2} \mathrm{O}, \mathrm{CO}, \mathrm{CO}_{2}, \mathrm{CH} 4$ and other small molecular. In the end, due to the introduction of $\mathrm{Fe}_{3} \mathrm{O}_{4}$ after modification, HPRs has a greater rate of carbon residue. The carbon residue rate of PRs is $33.9 \%$, and the carbon residue rate of HPRs is $39.1 \%$. Thermal gravimetric analysis shows that HPRs can be used below $120^{\circ} \mathrm{C}$, meeting the physical condition of adsorbing conventional oily substances.

Figure 2: The FT-IR spectra of PRs(a) and HPRs (b) 


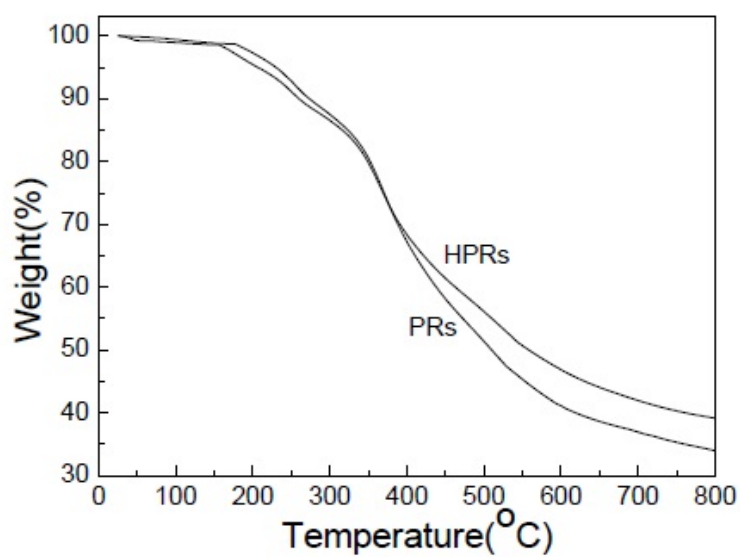

Figure 3: The TGA curves of PRs and HPRs

\subsection{SEM micrographs of HPRs}

The morphology of PRs and HPRs was characterized by using SEM. Fig. 4 is the SEM micrographs of PRs and HPRs, respectively. It can be seen that the HPRs surface is rough and irregular, which is beneficial to the adsorption of oily substances. Because of the removal of dispersed phase, PRs and HPRs exhibit three-dimensional interconnected pore structures and the diameter of open-cell is ranging from $2 \mu \mathrm{m}$ to $4 \mu \mathrm{m}$ (the average diameter is around $3 \mu \mathrm{m}$ ). The diameter of the interconnecting pore (from $0.3 \mu \mathrm{m}$ to $1.2 \mu \mathrm{m}$ ) is less than the diameter of open-cell. The opencell and interconnecting pore structure, which benefits to adsorb oily substances, increase the specific surface area of the materials and promotes the flow of the liquid.

Compared with the SEM micrographs of PRs and HPRs, spherical particles on the surface of HPRs are $\mathrm{Fe}_{3} \mathrm{O}_{4}$ particles. From the close-up view of HPRs, it can be observed that $\mathrm{Fe}_{3} \mathrm{O}_{4}$ particles which have a single size of about $600 \mathrm{~nm}$ are attached to the surface of HPRs. The prepared HPRs can be attracted by the external magnetic field, which indicates that the $\mathrm{Fe}_{3} \mathrm{O}_{4}$ particles have been successfully attached to the surface of the HPRs by polydopamine.

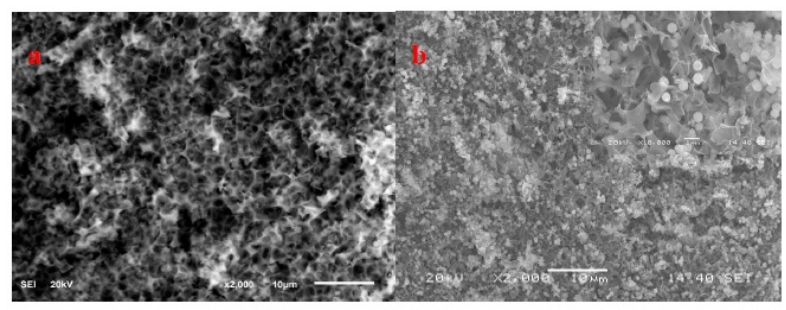

Figure 4: SEM micrographs of (a) PRs and (b) HPRS

\subsection{Energy spectrum analysis (EDS)}

The energy spectrum diagrams of PRs (a), NPRs (b) and HPRs(c) are shown in the Fig. 5.

The main elements of the unmodified PRs and NPRs that are obtained by reacting PRs with 1-dodecanethiol and $\mathrm{Fe}_{3} \mathrm{O}_{4}$ particles are only $\mathrm{C}$ and $\mathrm{O}$, which come from porous resin. As for HPRs that are obtained by reacting PRs with 1-dodecanethiol, dopamine hydrochloride and $\mathrm{Fe}_{3} \mathrm{O}_{4}$ particles, $\mathrm{Fe}$ and $\mathrm{S}$ elements also appear except $\mathrm{C}$ and $\mathrm{O}$ elements. From the result of the energy spectrum, it can be seen that the Fe and $\mathrm{S}$ elements do not appear in NPRs, suggesting that 1-dodecanethiol and $\mathrm{Fe}_{3} \mathrm{O}_{4}$ particles have not been modified successfully onto the surfaces of NPRs. This implies that the existence of dopamine hydrochloride is essential for the modification reaction. The self-polymerization of dopamine hydrochloride leads to that 1-dodecanethiol and $\mathrm{Fe}_{3} \mathrm{O}_{4}$ particles were anchored successfully onto the surfaces of HPRs.

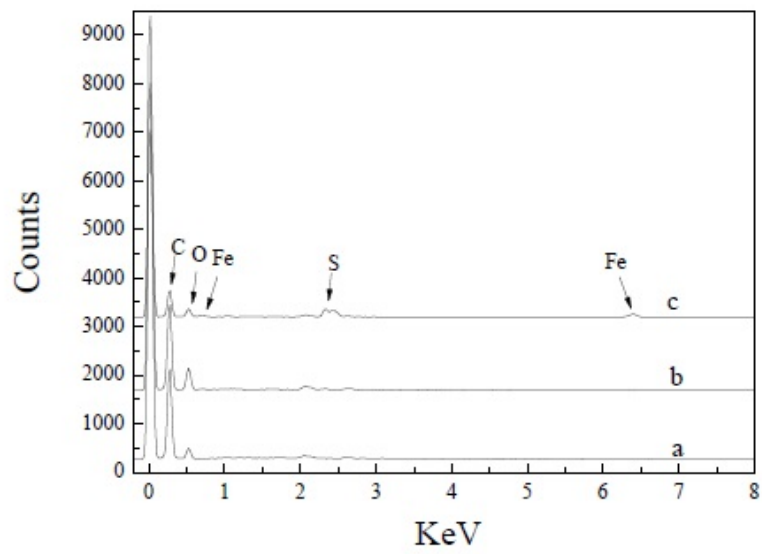

Figure 5: The energy spectrum diagrams of PRs(a), NPRs(b) and HPRs (c)

\subsection{Contact angle test and analysis}

The water contact angles of PRs and HPRs are shown in Fig. 6. The water contact angle of the PRs is $88.29^{\circ}$ and the contact angles of the new HPRs, HPRs- 1 and HPRs5 are $131.72^{\circ}, 123.60^{\circ}, 122.15^{\circ}$ respectively, indicating that PRs were successfully modified and the prepared HPRs had good hydrophobicity. It can be found from the figure that the contact angle of the HPRs decreases from $131.72^{\circ}$ to $123.60^{\circ}$ after one recycling experiment. The reason may be that during the recycling process, a small amount of combined 1-dodecanethiol falls off from the surface of the 
HPRs, reducing the hydrophobicity of the HPRs. After five recycling experiments, the decrease of the contact angle is not obvious and it is still more than $120^{\circ}$, which suggests that the structure of HPRs material is stable and HPRs can be used repeatedly.

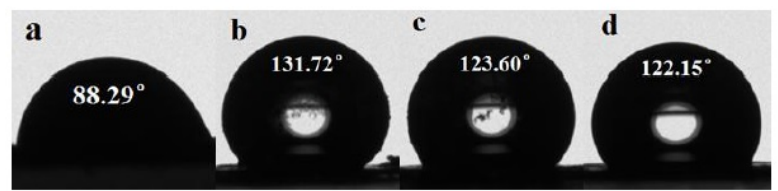

Figure 6: The water contact angles of the materials PRs (a), HPRs (b), HPRs-1: HPRs after one recycling experiment (c), HPRs-5 : HPRs after five recycling experiments (d)

\section{8 $\mathrm{N}_{2}$ adsorption-desorption isotherms}

Fig. 7 shows $\mathrm{N}_{2}$ adsorption-desorption isotherms of the HPRs. It shows that the adsorption isotherm of HPRs belongs to type IV adsorption isotherm. At the beginning, it is mainly single molecule layer adsorption, followed by multi-molecular layer adsorption and capillary condensation occurs in the medium pressure area. From the figure it can be seen that the range of hysteresis loop is wide and hysteresis loop starts from the low pressure area, indicating that a large number of pores appear in HPRs and the sizes of the pores are not uniform.

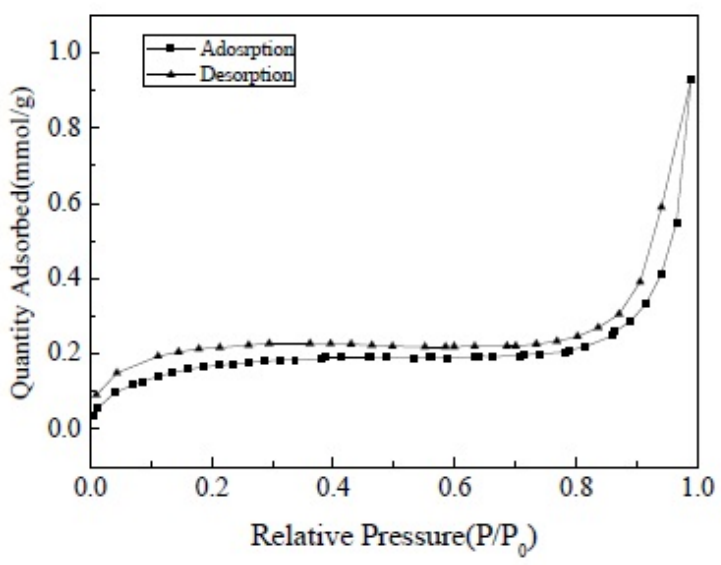

Figure 7: The N2 adsorption-desorption isotherm of the HPRs

The porous parameters of HPRs, HPRs-1 and HPRs5 are listed in Table 3. The BET specific surface area $\left(15.06 \mathrm{~m}^{2} / \mathrm{g}\right)$ and pore volume $\left(0.0349 \mathrm{~cm}^{3} / \mathrm{g}\right)$ of the HPRs1 are higher than those of the HPRs (BET specific surface area $\left(12.06 \mathrm{~m}^{2} / \mathrm{g}\right)$ and pore volume $\left.\left(0.0314 \mathrm{~cm}^{3} / \mathrm{g}\right)\right)$. This may be due to the closed pores are dredged after adsorption of toluene once.

However, after five times recycles, the specific surface area and pore volume are reduced. After one recycling experiment and five recycling experiments, the pore diameter reduced from $11.26 \mathrm{~nm}$ to $9.72 \mathrm{~nm}$ and $9.64 \mathrm{~nm}$, which may be caused by the incomplete removal of toluene which blocks the channels.

\subsection{The change of the oil adsorption rate with the time}

HPRs is an oil adsorbing material that has adsorption ability because of van der Waals force between HPRs and oil molecules. During the adsorption process, oil molecules spread to the surface of the HPRs by molecular diffusion and then oil molecules are adsorbed by the lipophilic groups on the HPRs. At the beginning, the oil adsorption rate is fast, but with the increasing of time, the oil adsorption rate slows down. The changes of the oil adsorption rate with the time for different oily substances are shown in Fig. 8. The oil adsorption rate increases rapidly during the first one minute and then it increases slowly over the time. Different types of oily substances are examined in the experiments and the results are similar. The oil adsorption rates within 1 minute for engine oil, bean oil, cyclohexane, acetone, gasoline and toluene are $9.551 \mathrm{~g} / \mathrm{g}, 9.360 \mathrm{~g} / \mathrm{g}$, $8.252 \mathrm{~g} / \mathrm{g}, 10.773 \mathrm{~g} / \mathrm{g}, 8.398 \mathrm{~g} / \mathrm{g}$ and $9.956 \mathrm{~g} / \mathrm{g}$, respectively. The oil adsorption rate reaches more than $80 \%$ of the highest oil adsorption rate in the first minute. With the increasing of time, the oil adsorption rate increases, while the increment is not very obvious. Eventually, the oil adsorption rates for engine oil, bean oil, cyclohexane, acetone, gasoline and toluene are $10.444 \mathrm{~g} / \mathrm{g}, 10.022 \mathrm{~g} / \mathrm{g}, 9.077 \mathrm{~g} / \mathrm{g}$, $13.432 \mathrm{~g} / \mathrm{g}, 10.787 \mathrm{~g} / \mathrm{g}$ and $11.638 \mathrm{~g} / \mathrm{g}$. This suggests that the HPRs can quickly adsorb oily substances, which makes it easy to remove oily substances in practical application.

\subsection{The oil adsorption rate of PRs and HPRs for different oily substance}

The oil adsorption rates of PRs and HPRs for different oils are shown in Fig. 9. It can be seen that the oil adsorption rates of HPRs with hydrophobic modification for different oily substances are higher than that of unmodified PRs. The oil adsorption rate of HPRs for acetone is the highest, followed by for toluene, gasoline, engine oil and bean oil, and the oil adsorption rate of HPRs of cyclohex- 
Table 3: The porous parameters of HPRs, HPRs-1 and HPRs-5

\begin{tabular}{lccc}
\hline \multicolumn{1}{c}{ Samples } & $\mathrm{SBET}\left(\mathrm{m}^{2} / \mathrm{g}\right)$ & $\mathrm{Vt}_{\left(\mathrm{cm}^{3} / \mathrm{g}\right)}$ & $\mathrm{DBJH}(\mathrm{nm})$ \\
\hline HPRs & 12.06 & 0.0314 & 11.26 \\
HPRs-1 & 15.06 & 0.0349 & 9.72 \\
HPRs-5 & 13.33 & 0.0285 & 9.64
\end{tabular}

Note: SBET is the BET specific surface area. $\mathrm{V}_{t}$ is the total pore volume determined the relative pressure of 0.99 . DBJH is mean pore diameter of $\mathrm{BJH}$ calculated by the desorption branches of the nitrogen sorption isotherms.

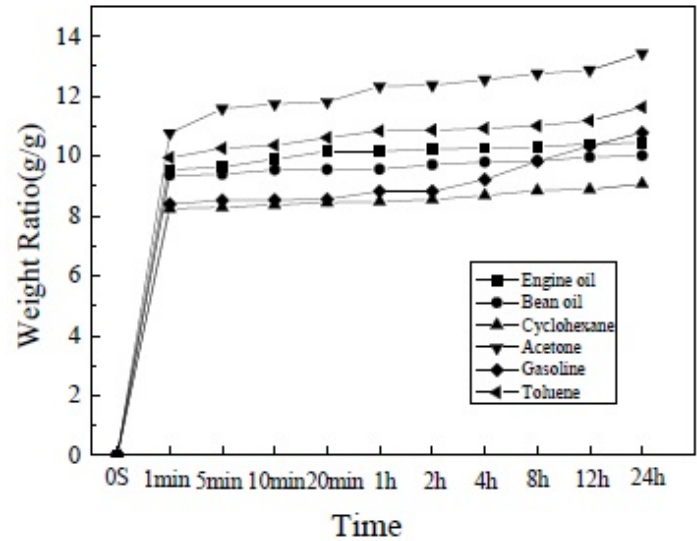

Figure 8: The changes of the oil adsorption rate with the time for different oily substances

ane is the lowest. After modification, the oil adsorption rate for acetone increases from $9.048 \mathrm{~g} / \mathrm{g}$ to $13.432 \mathrm{~g} / \mathrm{g}$. HPRs have higher oil adsorption rate for small molecule oily substances. But for engine oil, bean oil, gasoline and other long-chain oily substances, the oil adsorption rate decreases because the long-chain oily substances are not easy to enter the channels of the HPRs.

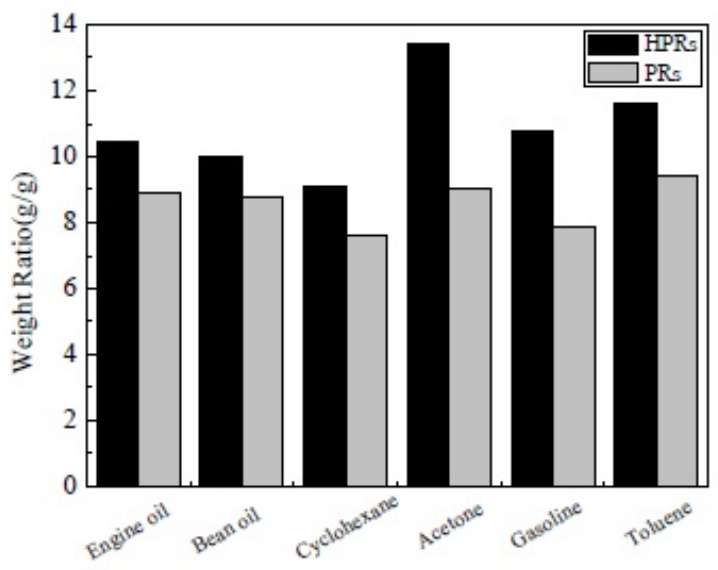

Figure 9: The oil adsorption rate of PRs and HPRs for different oils

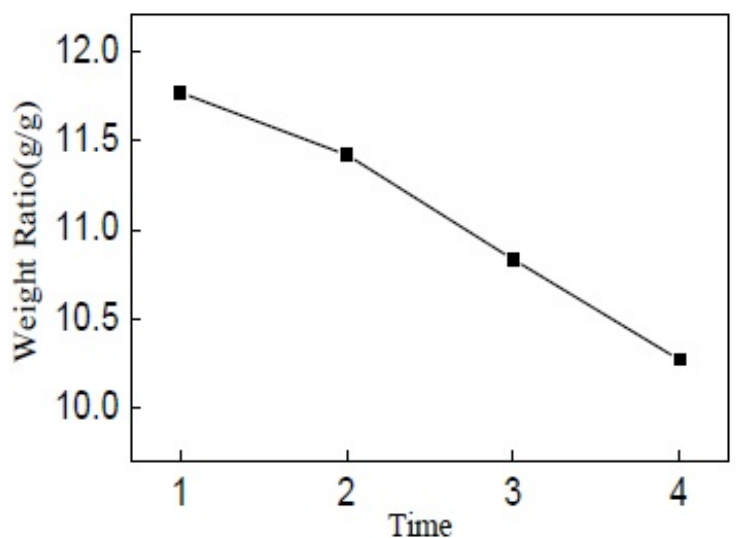

Figure 10: The oil adsorption rates of the freshly prepared and the recycled HPRs for toluene

The oil retention rates of HPRs for different oils are shown on Table 4. HPRs have a high oil retention rate for nonvolatile and high viscosity oily substances. On the contrary, HPRs has a low oil retention rate for volatile oils and small viscosity oily substances.

\subsection{Recovery performance of HPRs}

The oil adsorption rates of the freshly prepared and the recycled HPRs for toluene are shown in Fig. 10. According to the result of Fig. 10, the oil adsorption rate of the freshly prepared HPRs is $11.765 \mathrm{~g} / \mathrm{g}$. The oil adsorption rate reduces to $11.436 \mathrm{~g} / \mathrm{g}$ after one recycling experiment. With the increase of recycling times, the oil adsorption rate reduces may be due to the destruction of the three-dimensional interconnected pore structure and 1-dodecanethiol falling off from the surfaces of HPRs in the process of oil adsorption and regeneration. However, the oil adsorption rate of HPRs after three cycles is still $87.32 \%$ of that of the freshly prepared HPRs. It shows that HPRs maintains a good stability. 
Table 4: The oil retention rate of HPRs for different oily substances

\begin{tabular}{lclllll}
\hline $\begin{array}{l}\text { Products } \\
\begin{array}{l}\text { Oil retention } \\
\text { rate } / \%\end{array}\end{array}$ & Oil & Bean oil & Cyclohexane & Acetone & Gasoline & Toluene \\
\hline
\end{tabular}

\section{Conclusion}

1. PRs were prepared by HIPEs template method and then were modified by dopamine hydrochloride, 1dodecanethiol and $\mathrm{Fe}_{3} \mathrm{O}_{4}$ particles to achieve HPRs. The orthogonal experiments showed that the optimum technological parameters are: water-oil ratio of 1:5 and the dosages of 1-dodecanethiol, dopamine hydrochloride, $\mathrm{Fe}_{3} \mathrm{O}_{4}$ particles of $40 \mathrm{~g} / \mathrm{g}, 10 \%, 20 \%$ of PRs mass, respectively.

2. The surfaces of the material are grafted with 1dodecanethiol and $\mathrm{Fe}_{3} \mathrm{O}_{4}$ particles through polydopamine to achieve hydrophobicity and magnetic property. The water contact angle of HPRs can reach $130^{\circ}$ or more and they are easy to recycle under magnetic field. The thermal stability of HPRs is good under $120^{\circ} \mathrm{C}$, thus it is very suitable to adsorb oily substances under room temperature.

3. Compared with the unmodified PRs, the oil adsorption rate of HPRs increased obviously. HPRs have higher oil adsorption rate for small molecule oily substances and high oil retention rate for nonvolatile and high viscosity oily substances. They can quickly adsorb oily substances, and the oil absorption rate in the first minute can reach more than $80 \%$ of the highest oil adsorption rate.

4. The oil adsorption rate for toluene can reach $11.765 \mathrm{~g} / \mathrm{g}$ and the oil retention rate is $86.43 \%$. When HPRs are recycled three times, the water contact angle is still more than $120^{\circ}$, and the adsorption rate can still reach $87.32 \%$ of the first-time oil adsorption rate.

Acknowledgements: This work was financially supported by the National Natural Science Foundation of China (no.21407064), Doctoral Innovation Fund of Jiangsu University of Science and Technology (no.635211202).

\section{References}

[1] A. G. Dedov, E. E. Belousova, P. B. Kascsheyeva, E. I. Belyaeva, E. O. Omarova, E. C. Lobakova, E. A. Ivanova, R. K. Idialtulov, A. V. Genis and V. M. Buznik, Theoretical Foundations of Chemical Engineering, 2014, 48, 706-715.
[2] C. F. Wang and S. J. Lin, ACS Applied Materials \& Interfaces, 2013, 5, 8861-8864.

[3] L. Xiong, W. Min, C. Wang, C. Cheng and X. Wang, ACS Appl Mater Interfaces, 2014, 6, 15272-15282.

[4] X. Gao, L. P. Xu, Z. Xue, L. Feng, J. Peng, Y. Wen, S. Wang and X. Zhang, Advanced Materials, 2014, 26, 1771-1775.

[5] C. R. Crick, J. A. Gibbins and I. P. Parkin, Journal of Materials Chemistry A, 2013, 1, 5943-5948.

[6] A. Li, H. X. Sun, D. Z. Tan, W. J. Fan, S. H. Wen, X. J. Qing, G. X. Li, S. Y. Li and W. Q. Deng, Energy \& Environmental Science, 2011, 4, 2062-2065.

[7] A. Atta, W. Brostow, H. Hagglobland, A. R. Hasan and J. Perez, RSC Advances, 2013, 3, 25849-25857.

[8] D. Wu, F. Xu, B. Sun, R. Fu, H. He and K. Matyjaszewski, Chemical Reviews, 2012, 112, 3959.

[9] C. R. Langford, D. W. Johnson and N. R. Cameron, Macromolecular Rapid Communications, 2015, 36, 834-839.

[10] U. H. F. Bunz, Advanced Materials, 2006, 18, 973-989.

[11] C. Chi, H. Xu, K. Zhang, Y. Wang, S. Zhang, X. Liu, X. Liu, J. Zhao and Y. Li, Materials Science \& Engineering B, 2015, 194, 62-67.

[12] H. Gao, Y. Peng, J. Pan, J. Zeng, C. Song, Y. Zhang, Y. Yan and W. Shi, RSC Advances, 2014, 4, 43029-43038.

[13] Y. Wu, T. Zhang, Z. Xu and Q. Guo, Journal of Materials Chemistry A, 2015, 3, 1906-1909.

[14] J. Zeng, Y. Peng, J. Pan, H. Gao, R. Wu, Y. Yin and Y. Yan, Chemical Engineering Journal, 2015, 266, 1-11.

[15] X. Wang, X. Chen, Y. Peng and J. Pan. RSC Advances, 2019, 9(13):7228-7237.

[16] T. Zhang and M. S. Silverstein, Polymer, 2017, 126.

[17] C. Chen, A. M. Eissa, T. L. Schiller and N. R. Cameron, Polymer, 2017, 126.

[18] S. Huš, M. Kolar and P. Krajnc, Designed Monomers \& Polymers, 2015, 18, 698-703.

[19] X. Huang, Y. Peng, J. Pan, W. Zhang, W. Zhou, H. Zhu and S. Liu, Journal of Applied Polymer Science, 2017, 134.

[20] C. W. Damouny and M. S. Silverstein, Polymer, 2016, 82, 262273.

[21] T. Gitli and M. S. Silverstein, Polymer, 2011, 52, 107-115.

[22] F. D. Balacianu, A. C. Nechifor, R. Bartos, S. I. Voicu and G. Nechifor, Optpelectronics and Advanced Materials-rapid Communications, 2009, 3, 219-222.

[23] D. P. Nair, M. Podgórski, S. Chatani, T. Gong, W. Xi, C. R. Fenoli and C. N. Bowman, Cheminform, 2014, 45, 724-744.

[24] A. Turco, C. Malitesta, G. Barillaro, A. Greco, A. Maffezzoli and E. Mazzotta, Journal of Materials Chemistry A, 2015, 3, 17685 17696.

[25] Y. Duan, F. Bian and H. Huang, Polymers for Advanced Technologies, 2016, 27, 228-234. 